Angular Distribution for ${ }^{56} \mathrm{Fe}\left(\pi^{+}, \pi^{-}\right)^{56} \mathrm{Ni}(\mathrm{DLAS})$

R. Gilman

University of Pennsylvania, Philadelphia, PA 19104 and

Argonne National Laboratory, Argonne, IL 60499-1819

\section{L. Morris}

Los Alamos National Laboratory, Los Alamos, NM 87545

G. R. Burleson and Kalvir S. Dhuga

New Mexico State University, Las Cruces, NM 88008

M. Burlein, H. T. Fortune, and J. D. Zumbro University of Pennsylvania, Philadelphia, PA 19101

M. A. Machuca, C. Fred Moore, S. Mordechai*, D. S. Oakley, and M. J. Smithson

University of Texas at Austin, Austin, TX r8712

\author{
D. L. Watson \\ University of York, York, YO1 5DD, UK \\ and \\ Los Alamos National Laboratory, Los Alamos, NM 87515
}

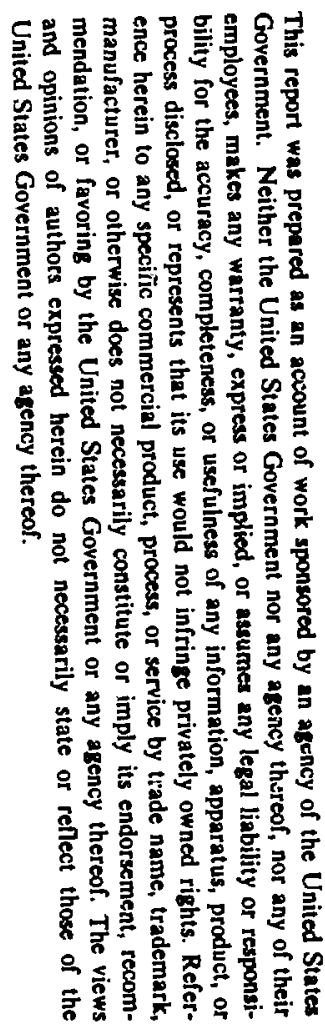

\title{
ABSTRACT
}

We have measured a $\left(\pi^{+}, \pi^{-}\right)$angular distribution for the transition to the double isobaric analog state of a $T=2$ target nucleus, ${ }^{56} \mathrm{Fe}$, at $\mathrm{T}=180 \mathrm{MeV}$. The data are compared to messurements on $T=1$ targets and to theoretical predictions. The angular dependence is different from that observed in measurements on $T=1$ targets. Some features of the data are reproduced by the theoretical predictions, but significant discrepancies remain.

PACS numbers: 21.10.Hw, 24.10.Ht, 24.30.Eb, 25.80.Fm

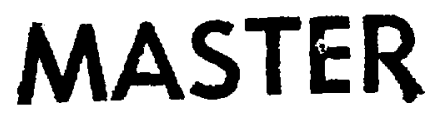


All the published comparisons of data with theory for $\left(\pi^{+}, \pi^{-}\right)$double charge exchange (DCX) angular distributions leading to the double isobaric analog state (DIAS) have been for $T=1$ states, primarily because of the difficulty of $\left(\pi^{+}, \pi^{-}\right)$ measurements on $T>1$ targets. For transitions to the DLAS the cross section decreases with mass as $(N-Z)(N-Z-1) A^{-10 / 3}$, and for $T>1$, the DLAS is an excited state that must be extracted from a continuum background. Thus, the validity of theories for $T>1$ targets has remained untested.

For $T=1$ nuclei and energies near $\mathrm{T}_{\pi}=164 \mathrm{MeV}$, the angular distribution data exhibit a deep minimum (at least an order of magnitude decrease of the cross section) at an angle several degrees smaller than the position of a minimum predicted by simple models (sequential charge exchange through the free pionnucleon amplitude $)^{1,2,3}$. The theories of Johnson and Siciliano ${ }^{4,5}$ and of Liu ${ }^{6}$ successfully describe a majority of the angular distributions at $T_{\pi}=164 \mathrm{MeV}$, by including second-order terms in the $\pi$-A optical potential that account for higher order processes. At higher energies, $T_{\pi} \geq 230 \mathrm{MeV}$, the simpler models adequately describe the existing data ${ }^{1,2}$.

Fig. 1 shows predictions of angular distributions for ${ }^{56} \mathrm{Fe}\left(\pi^{+}, \pi^{-}\right)^{56} \mathrm{Ni}$ (DLAS) at $\mathrm{T}_{\pi}=180 \mathrm{MeV}$, calculated using the code PIESDEX ${ }^{4}$ with Hartree-Fock (Skyrme III effective interaction) neutron, proton, and excess neutron densities ${ }^{7}$. The dotted curve includes no second-order optical-potential terms. Thus it represents sequential scattering through the intermediate analog state. Although the second-order potential parameters are energy dependent (but $A$ independent), 
the paucity of single- and double-charge-exchange data at $T_{\pi}=180 \mathrm{MeV}$ precluded a unified analysis along the procedure described by Greene et al. ${ }^{5}$ Thus, we have used the $164-\mathrm{MeV} \rho^{2}$ optical-potential parameters (coefficients of the higher order potential terms proportional to $\rho^{2},(\Delta \rho)^{2}$, and $\rho \Delta \rho$, where $\rho$ and $\Delta \rho$ are the nuclear and excess neutron density, see Table I) in the two other calculations. The dashed curve is a second-order calculation for the $T=1 \operatorname{target}{ }^{54} \mathrm{Fe}$, and the solid curve is the same calculation for the ${ }^{56} \mathrm{Fe}$ target. The striking feature of the calculations is the difference in the position of the minima: at $\theta=$ 22,14 and $30^{\circ}$. Thus, for $T=2$ target nuclei, the position of the first minimum is predicted to be at a much larger angle than that predicted by the lowest order calculation, while for $T=1$ nuclei, the reverse is true ${ }^{1,2,8}$. (A linear extrapolation of the second-order parameters between results at $164 \mathrm{MeV}$ (ref. 5) and 230 $\mathrm{MeV}$ (ref. 9) does not change the results appreciably.) The change in the position of the minimum results from the isospin dependence of the second-order $\pi-A$ interaction -- which describes higher order processes such as delta-nucleus interactions, pion absorption, and two-particle correlations - contributing to DCX transitions to the DIAS.

We present here the first measurement of an angular distribution on a $T>1$ target, performed as a test of the above predictions, the validity of the unified analysis of ref. 5, and the formalism of Johnson and Siciliano ${ }^{4}$. There exist two angular distribution measurements at $\mathrm{T}_{\pi}=180 \mathrm{MeV}$ for $T=1$ target nuclei, ${ }^{18} \mathrm{O}\left(\pi^{+}, \pi^{-}\right)^{18} \mathrm{Ne}$ (DIAS) (ref. 2) and ${ }^{42} \mathrm{Ca}\left(\pi^{+}, \pi^{-}\right){ }^{42} \mathrm{Ti}$ (DLAS) (ref. 8), to which we can compare our measurement. In this letter, we report differential 
cross sections for ${ }^{56} \mathrm{Fe}\left(\pi^{+}, \pi^{-}\right)^{56} \mathrm{Ni}\left(\mathrm{DLAS}, \mathrm{E}_{\mathrm{x}}=0.90 \pm 0.05 \mathrm{MeV}\right)$, $5 \leq \theta \leq 30^{\circ}$, at $\mathrm{T}_{\pi}=180 \mathrm{MeV}$. A more complete description of the measurements and other data will be reported later ${ }^{10}$.

The measurements were made at the Clinton P. Anderson Meson Physics Facility (LAMPF) with the energetic pion channel and spectrometer (EPICS). Descriptions of the channel, spectrometer, and modifications for forward aagle DCX measurements have been presented elsewhere ${ }^{11}$.

The DCX data were normalized by measuring yields for ${ }^{1} \mathrm{H}\left(\pi^{+}, \pi^{+}\right)^{1} \mathrm{H}$ at $\theta=40^{\circ}$, and comparing them to cross sections based on the phase shifts of Rowe, Salomon and Landau ${ }^{12}, A^{1} \mathrm{H}\left(\pi^{+}, \pi^{+}\right)^{1} \mathrm{H}$ angular distribution showed that any angle-dependent systematic errors are less than $2 \%$.

Cross sections were extracted for the DIAS with the code NEWFIT, written by one of us (CLM). NEWFIT is an interactive line-shape fitting code that uses the CERN MINUIT optimization package. The fitted line shape was a Landau distribution folded with a Gaussian ( $400 \mathrm{keV}$ FWHM) to fit elastic scattering spectra. The broadening of the lineshape accounts for instrumental resolution and multiple scattering in the target. With the momentum-dispersed EPICS pion beam, the incident energy is derived from the projected interaction position at the scattering target. Multiple scattering decreases the iesolution of the projection back to the target. The resulting full width of $1.0 \mathrm{MeV}$ at one-half maximum (2.4 $\mathrm{MeV}$ at one-tenth maximum) was caused mostly by straggling in the target $\left(2.38 \mathrm{gm} / \mathrm{cm}^{2}\right.$ cold rolled steel). 
There are very few background counts in the region of the ${ }^{56} \mathrm{Ni}$ (DIAS) from scattering from other isotopes. We expect that the worst case would be for ${ }^{54} \mathrm{Fe}\left(\pi^{+}, \pi^{-}\right)^{54} \mathrm{Ni}(\mathrm{gs})$, which would be centered $0.44 \mathrm{MeV}$ above the ${ }^{56} \mathrm{Ni}$ (DLAS) in excitation energy, with approximately $6 \%$ of the intensity of the ${ }^{56} \mathrm{Ni}$ (DLAS) peak. Our data do not indicate a ${ }^{54} \mathrm{Ni}(\mathrm{gs})$ peak (much improved statistics would be needed to observe it), and thus we have made no explicit corrections for it. Other isotopes, due either to their more negative Q-values for the $\left(\pi^{+}, \pi^{-}\right)$reaction or to their small abundance, make a negligible contribution.

To extract yields at $180 \mathrm{MeV}$, the following procedure was used. A summed spectrum was created of all DCX data at all angles without any correction for relative normalizations or acceptance, (the acceptance varied by less than $3 \%$ across the fitted region of $\mathrm{E}_{\mathrm{x}}=5.0$ to $15.0 \mathrm{MeV}$ ). Three peaks plus an exponential background were required to fit the summed data well. Removing the nonDLAS peaks from the fit increases the $\chi^{2}$ with only a small change in the extracted cross sections. These results were used as initial values for fits at each angle. The variation in peak positions with angle was less than $100 \mathrm{keV}$ (the three peaks were fixed relative to each other). At $30^{\circ}$, no DLAS peak was visible. Without a constraint on the peak positions, the best fit extracted peak positions differed from those at other angles by about $0.5 \mathrm{MeV}$, which was considered unacceptably different. Thus, the peak positions at $30^{\circ}$ were constrained to be the summed spectrum results. A sample fit at $15^{\circ}$ is shown in Fig. 2.

The extracted excitation energy of the DLAS peak, $9.90 \pm 0.05 \mathrm{MeV}$, differs somewhat from our previous measurement ${ }^{13}\left(E_{x}=9.6 \pm 0.2 \mathrm{MeV}\right)$ with the 
same reaction at several energies between $T_{\pi}=164$ and $292 \mathrm{MeV}$. In the previous work, an inferior fitting algorithm was used, which accounts for the larger uncertainty in $E_{x}$. Our determination of the excitation energy is in good agreement with determinations from ${ }^{54} \mathrm{Fe}\left({ }^{3} \mathrm{He}, \mathrm{n}\right){ }^{56} \mathrm{Ni}$ and ${ }^{58} \mathrm{Ni}(\mathrm{p}, \mathrm{t}){ }^{56} \mathrm{Ni}$ measurements $^{14}$, which find $\mathrm{E}_{\mathrm{x}} \approx 8.87 \mathrm{MeV}$

The ${ }^{56} \mathrm{Fe}$ data are compared to the ${ }^{18} \mathrm{O}$ and ${ }^{42} \mathrm{Ca}$ measurements and PIESDEX calculations in Fig. 3. The ${ }^{56} \mathrm{Fe}$ differential cross section exhibits a forward angle peak that falls by a factor of approximately three to a relatively unvarying cross section in the region $15<\theta<25^{\circ}$. At $30^{\circ}$, the cross section has dropped by a factor of at least 1.5 from the $25^{\circ}$ value, and might indicate a minimum near $30^{\circ}$. The data are inconsistent with the presence of a deep minimum in the vicinity of $14^{\circ}$, as we would expect based on the ${ }^{18} \mathrm{O},{ }^{42} \mathrm{Ca}\left(\pi^{+}, \pi^{-}\right)^{18} \mathrm{Ne},{ }^{42} \mathrm{Ti}(\mathrm{DLAS})$ measurements, and the hypothesis that the angular distributions for $T=2$ targets would have similar features as those for $T=1$ targets. The data are also inconsistent with a minimum at $20^{\circ}$, as the first-order calculation predicts.

We have used the second-order parameters that were determined in the unified analysis of ref. 5 for the calculations shown. These results correctly predict the position of the minimum for ${ }^{18} \mathrm{O}$ and ${ }^{42} \mathrm{Ca}$. However, there are shortcomings for ${ }^{18} \mathrm{O}$ at larger angles, and the overall magnitude of the ${ }^{42} \mathrm{Ca}$ calculation is incorrect. Though the calculation for ${ }^{56}$ Fe correctly predicts a relatively low cross section near $\theta=30^{\circ}$, there remain significant differences in shape and magnitude between the data and the calculation (solid curve). 
More specifically, values of $\chi^{2}$ calculated with respect to the three calculations of Fig. 1 show that the best agreement is with the calculation for ${ }^{56} \mathrm{Fe}$ including the higher potential terms (solid curve), and the worst is with the simpler calculation (dashed curve). Renormalization of the calculations to best fit the data yields the same conclusions. Thus the $T=2$ data exhibit features unlike $\left(\pi^{+}, \pi^{-}\right)$measurements for $T=1$ nuclei at, or slightly above, $\mathrm{T}_{\pi}=164$ $\mathrm{MeV}$.

With respect to the phenomenology of DCX, the ${ }^{56} \mathrm{Fe}$ angular distribution at $\mathrm{T}_{\pi}=180 \mathrm{MeV}$ increases our knowledge. It exhibits neither the deep forward minimum characteristic of $T=1$ nuclei, nor a lowest-order shape as might be expected from a naive two-amplitude model ${ }^{15}$. A new type of behavior has been found. However, the lack of detailed agreement with the second-order prediction presented here is also not encouraging.

How serious is the disagreement? It is difficult to judge since the secondorder parameters are expected to be strongly energy dependent in the $\Delta_{33}$ reso nance region. We have successfully adjusted the magnitudes of isovector and isotensor terms to reproduce the ${ }^{56} \mathrm{Fe}$ angular distribution, but we have not been able to both qualitatively reproduce the shoulder (the unvarying cross section for $15<\theta<25^{\circ}$ ) in the ${ }^{56} \mathrm{Fe}$ data and retain a forward minimum in the ${ }^{18} \mathrm{O}$ and ${ }^{42} \mathrm{Ca} 180-\mathrm{MeV}$ angular distributions (within two or three degrees of the data) with the same set of parameters. We note that a shoulder is predicted ${ }^{10}$ for the $T=2$ nucleus ${ }^{44} \mathrm{Ca}$ at $164 \mathrm{MeV}$, but we have not been successful at using this 
as a starting point for adjusting the assumed ${ }^{56} \mathrm{Fe}$ nuclear structure to allow prediction of a shoulder. We conclude that the present phenomenology is inadequate, because the shoulder in the data is not reproduced. It is not, however, a fatal flaw, because elastic scattering and single charge exchange input does not exist. Also, the unified analysis phenomenology does successfully predict a difference in shapes between $T=1$ and $T=2$ target nuclei.

In conclusion, we have measured the angular distribution for $\left(\pi^{+}, \pi^{-}\right)$on a $T=2$ target nucleus. The data are inconsistent with a deep minimum at an angle smaller than $30^{\circ}$. Thus, the data are also inconsistent with the lowestorder calculation or with $\left(\pi^{+}, \pi^{-}\right)$measurements on $T=1$ target nuclei, where minima appear at smaller angles. We interpret this feature to be a characteristic difference between $T=1$ and $T=2$ measurements near the peak of the $\Delta_{33}$ resonance. These data and further measurements of this type will place constraints on theories of the pion-nucleus interaction.

This work has been supported in part by the U.S. Department of Energy, The Robert A. Welch Foundation, and the National Science Foundation. Argonne National Laboratory is supported by the U.S. Department of Energy Nuclear Physics Division under contract W-31-109-ENG-38. 


\section{REFERENCES}

1+1 Permanent address: Ben Gurion University of the Negev, Beer-Sheva, Israel.

1

R. Gilman, H. T. Fortune, J. D. Zumbro, Peter A. Seidl, C. Fred Moore, C. L. Morris, J. A. Faucett, G. R. Burleson, S. Mordechai, and Kalvir S. Dhuga, Phys. Rev. C33, 1082 (1986).

Peter A. Seidl, C. Fred Moore, S. Mordechai, R. Gilman, Kalvir S. Dhuga, H. T. Fortune, J. D. Zumbro, C. L. Morris, J. A. Faucett, and G. R. Burleson, Phys. Lett. 154B, 255 (1985).

Peter A. Seidl, Mark D. Brown, Rex R. Kiziah, C. Fred Moore, Helmut Baer, C. L. Morris, G. R. Burleson, W. B. Cottingame, Steven J. Greene, L. C. Bland, R. Gilman, and H. T. Fortune, Phys. Rev. C30, 973 (1983).

M. B. Johnson and E. R. Siciliano, Phys. Rev. C27, 1647 (1983).

S. J. Greene, C. J. Harvey, P. A. Seidl, R. Gilman, E. R. Siciliano, and Mikkel B. Johnson, Phys. Rev. C30, 2003 (1984).

6

L. C. Liu, Phys. Rev. C27, 1611 (1983).

M. Biener, H. Flocard, Nguyen Van Ghai, and P. Quentin, Nucl. Phys. A238, 29 (1975). 
8 K. K. Seth, in "Proceedings of the Tenth International Conference on Particles and Nuclei”, Nucl. Phys. A434, 287c (1985).

9 R. Gilman, M. B. Johnson, and P. A. Seidl (unpublished).

10 R. Gilman, P. A. Seidl, G. Rai, C. L. Morris, G. R. Burleson, Kalvir S. Dhuga, M. Burlein, H. T. Fortune, J. D. Zumbro, M. A. Machuca, C. Fred Moore, S. Mordechai, D. L. Watson, (unpublished).

11 H. A. Thiessen et al., Los Alamos Scientific Laboratory Report LA6663-MS (1977); S. J. Greene et al., Phys. Lett. 88B, 62 (1979); S. J. Greene, $\mathrm{PhD}$ thesis, The University of Texas at Austin, Los Alamos National Laboratory Report LA-8881-T (1981). G. Rowe, M. Salomon, and R. H. Landau, Phys. Rev. C18, 584 (1978). Peter A. Seidl, Rex R. Kiziah, Mark D. Brown, C. Fred Moore, C. L. Morris, Helmut Baer, Steven J. Greene, G. R. Burleson, W. B. Cottingame, L. C. Bland, R. Gilman, and H. T. Fortune, Phys. Rev. Lett. 50,1106 (1983).

14 D. Evers, W. Assmann, K. Rudolph, and S. J. Skorka, Nucl. Phys. A198, 268 (1972); H. Nann, and W. Benenson, Phys. Rev. C10, 1880 (1874).

15 R. Gilman, L. C. Bland, Peter A. Seidl, C. Fred Moore, C. L. Morris, Steven J. Greene, and H. T. Fortune, Nucl. Phys. A432, 610 (1985). 


\section{Table I}

Coefficients used for the $\rho^{2}$ terms (Jo 83) of the calculations of Fig. 1.

\begin{tabular}{|c|c|c|c|c|c|}
\hline Curve & $\begin{array}{c}\mathrm{T}_{\pi} \\
(\mathrm{MeV})\end{array}$ & $\begin{array}{c}\lambda_{0}^{(2)} \\
\left(\mathrm{fm}^{3}\right)\end{array}$ & $\begin{array}{c}\lambda_{1}^{(2)} \\
\left(\mathrm{fm}^{3}\right) \\
\end{array}$ & $\begin{array}{r}\lambda^{(2)} \\
\left(\mathrm{fm}^{3}\right)\end{array}$ & $\begin{array}{c}\text { Energy } \\
\text { Shift } \\
(\mathrm{MeV}) \\
\end{array}$ \\
\hline $\begin{array}{l}\text { otted } \\
\text { olid, dashed }\end{array}$ & $\begin{array}{l}180 \\
180\end{array}$ & $\begin{array}{l}0+0 \mathrm{i} \\
0.75+3.67 \mathrm{i}\end{array}$ & $\begin{array}{l}0+0 \mathrm{i} \\
7.71+15.5 \mathrm{i}\end{array}$ & $\begin{array}{l}0+0 \mathrm{i} \\
1.66+10.8\end{array}$ & $\begin{array}{l}0+0 i \\
35.0+0.3 i\end{array}$ \\
\hline
\end{tabular}




\section{FIGURE CAPTIONS}

Fig. $1\left(\pi^{+}, \pi^{-}\right)$calculations from the code PIESDEX ${ }^{4}$ at $T_{\pi}=180 \mathrm{MeV}$ for transitions to the DLAS. The dotted curve represents sequential scattering through the intermediate analog state, with no higher order processes. The solid curve represents a second-order calculation for ${ }^{56} \mathrm{Fe}$, with the strengths of the second-order potential taken from fits to $164 \mathrm{MeV}$ data $^{5}$ (see Table I). The dashed curve represents a calculation using the same second-order potential as for the solid curve, for the target nucleus ${ }^{54} \mathrm{Fe}$.

Fig. 2 An example of a fit to the excitation energy spectra at $15^{\circ}$ from the code NEWFIT. The lines represent the statistical uncertainty of the data. The curves show the three peaks and the exponential background that were used to parameterize the data. The DIAS is at $8.90 \pm 0.05$ $\mathrm{MeV}$, the identity of the peaks above and below the DIAS is ambiguous. The spectrum has not been corrected for spectrometer acceptance as a function of excitation energy.

Fig. 3 Data for ${ }^{18} \mathrm{O}\left(\pi^{+}, \pi^{-}\right)^{18} \mathrm{Ne}$ (DIAS) (crosses, ref. 2), ${ }^{42} \mathrm{Ca}\left(\pi^{+}, \pi^{-}\right)^{42} \mathrm{Ti}$ (DLAS) (open circles, ref. 8), and ${ }^{56} \mathrm{Fe}\left(\pi^{+}, \pi^{-}\right)^{56} \mathrm{Ni}(\mathrm{DLAS})$ (squares, this work) at $\mathrm{T}_{\pi}=180 \mathrm{MeV}$ are compared to PIESDEX ${ }^{4}$ calculations for which the second-order interaction parameters are identical to those of the solid curve in Fig.

1. The data and calculations for the ${ }^{18} \mathrm{O}$ and ${ }^{42} \mathrm{Ca}$ targets have been 
multiplied by 100 . The dashed curve is the same as the dashed curve in Fig. 1, multiplied by the additional factor of 6 (ratio of the statistical factor $(N-Z)(N-Z-1) / 2$ for ${ }^{54} \mathrm{Fe}$ and $\left.{ }^{56} \mathrm{Fe}\right)$. 


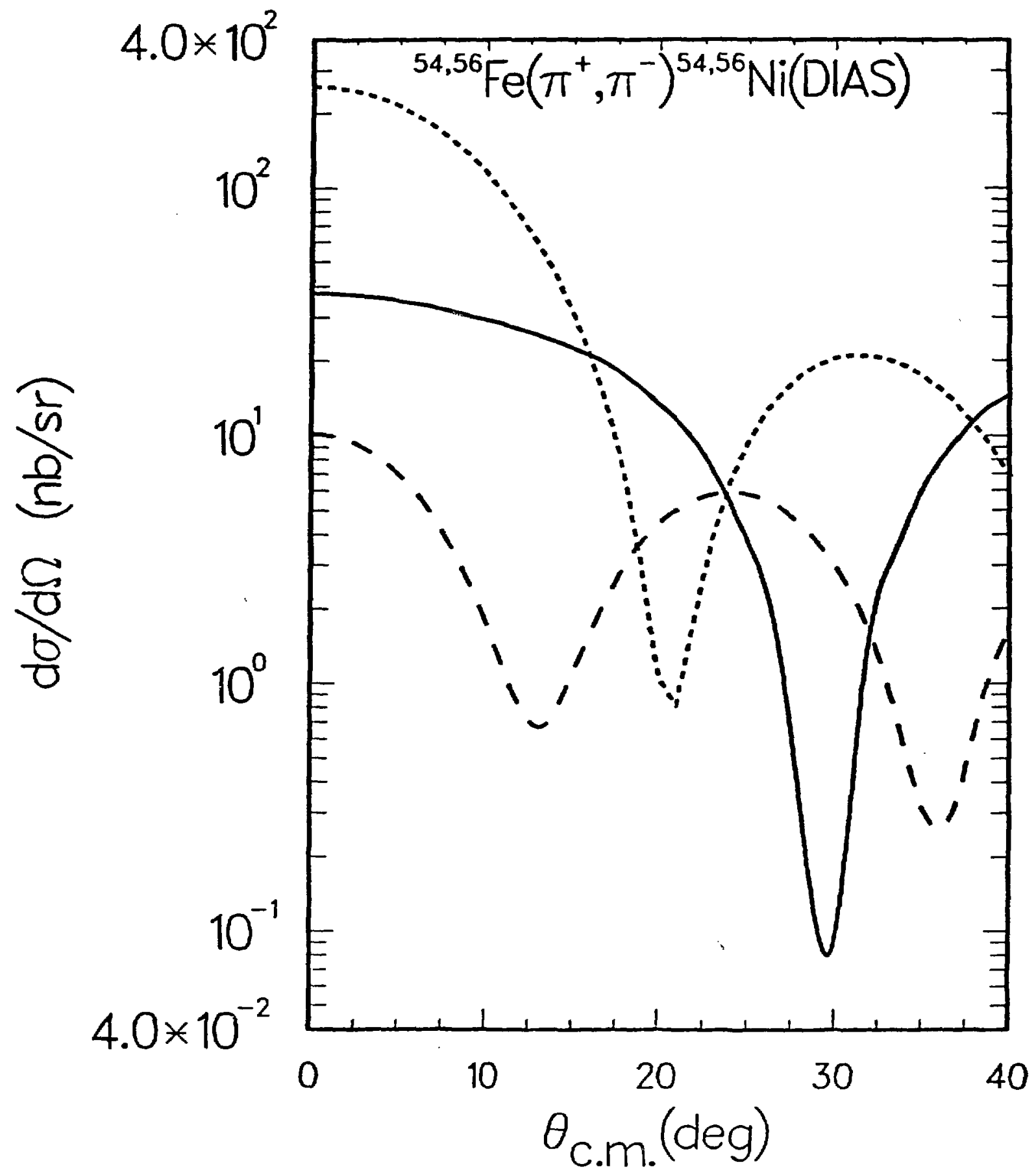

Fig. 1 


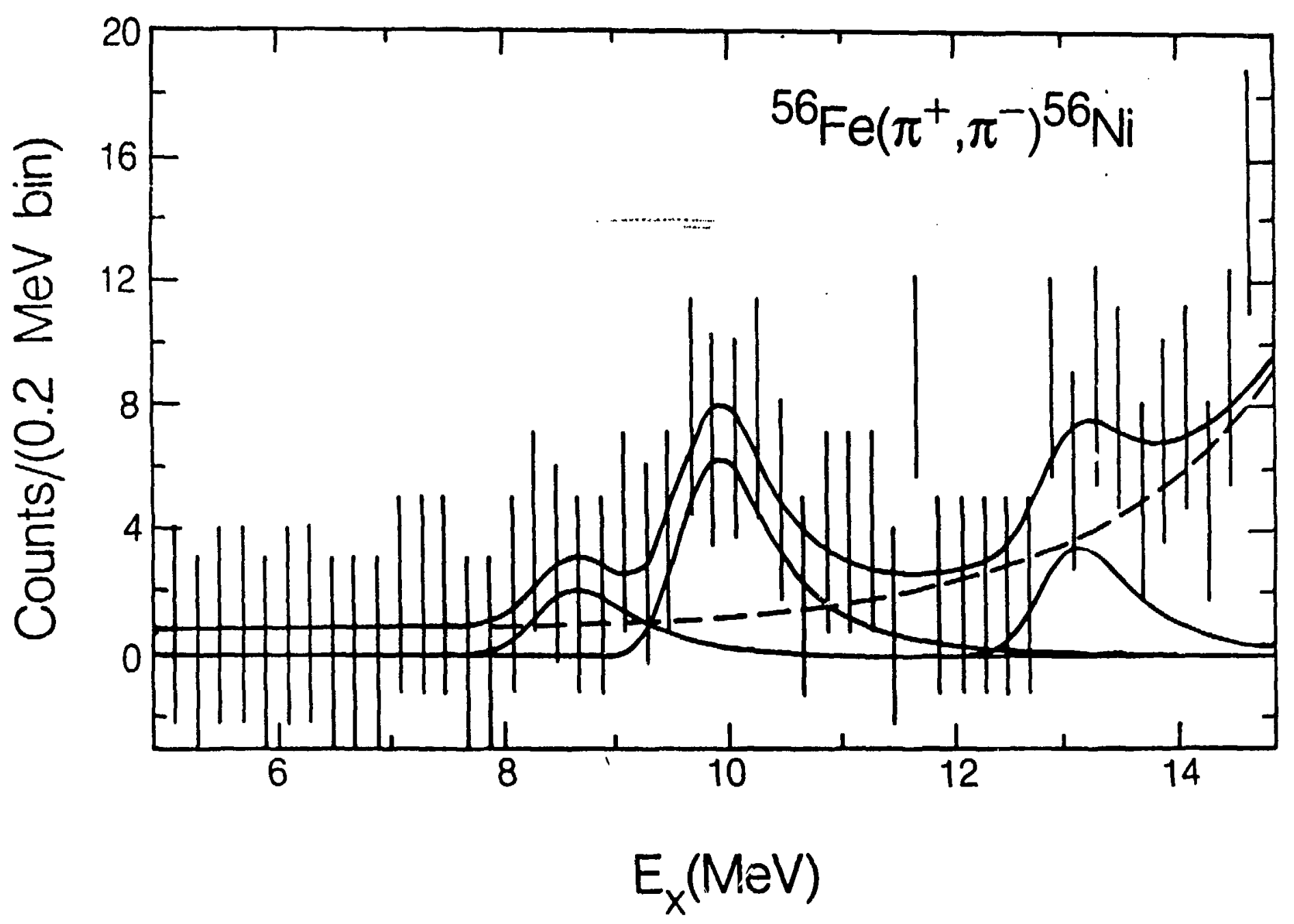

XBL 872.9566

Fig. 2 


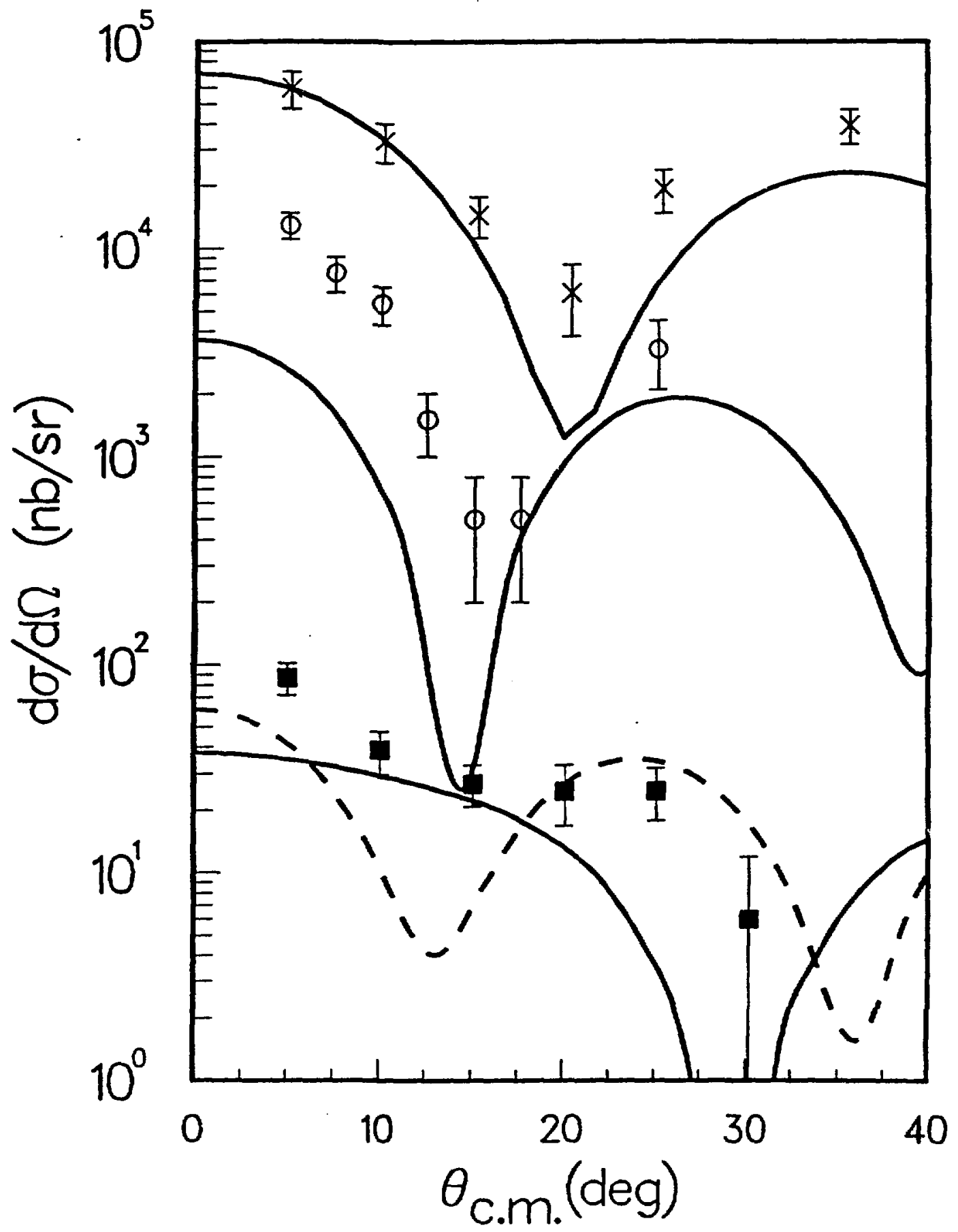

FIg. 3 